\title{
Publicaciones de autores chilenos en revistas quirúrgicas durante los últimos diez años*
}

\author{
Drs. JAVIER MORAGA C. ${ }^{1}$, RICARDO CARTES-VELÁSQUEZ. ${ }^{2}$, CARLOS MANTEROLA D. ${ }^{1,2,3}$, \\ SEBASTIÁN URRUTIA V. ${ }^{3}$ GRUPO MINCIR (METODOLOGÍA E INVESTIGACIÓN EN CIRUGÍA).
} 1 Programa de Magíster en Ciencias Médicas, Mención Cirugía, Universidad de La Frontera.
2 Programa de Doctorado en Ciencias Médicas, Universidad de La Frontera.
3 Departamento de Cirugía y Traumatología. Facultad de Medicina, Universidad de La Frontera.
Temuco, Chile.

\begin{abstract}
Analysis of scientific publications by Chilean surgeons

Background: The number and quality of publications is an indirect measure of the capacity of an individual, institution or country to generate knowledge. Aim: To assess the number of publications of Chilean surgeons, registered in the Institute for Scientific Knowledge (ISI). Material and Methods: A search was conducted in the ISI platform for publications generated by Chilean surgeons in the last ten years. The number of publications and citations, the journals in which the publications appeared and nationality of coauthors, were analyzed. Results: Three hundred and eighty papers were identified. Of these, 333 (88\%) were published in the period 2006-2010. The papers were cited in 1.946 opportunities (impact factor of 5.12 and h-index of 21). Thirty three percent of papers were published in Revista Chilena de Cirugía, $8 \%$ in Obesity Surgery and $4 \%$ in the World Journal of Surgery. Thirty four percent of papers came from the University of Chile, 21\% from Catholic University and 8\% from Universidad de la Frontera. Conclusions: The number of publications of Chilean surgeons is experiencing an increase in the last years.

Key words: Surgical Procedures, Operative [MeSH], Bibliometrics [MeSH], ISI Web of knowledge, Surgery $[\mathrm{MeSH}]$.
\end{abstract}

\section{Resumen}

Introducción: Caracterizar la producción científica personal, institucional o nacional es una necesidad para comprender y mejorar la generación de conocimiento. El objetivo de este estudio es definir el perfil bibliométrico de las publicaciones ISI, relacionadas con la cirugía chilena en el período 2001-2010. Material y Método: Estudio bibliométrico. Se realizó una búsqueda en la plataforma ISI Web of Knowledge de Thomson Reuters respecto de la productividad científica de la cirugía chilena en los últimos 10 años. Las variables analizadas fueron número de publicaciones, citaciones de artículos chilenos por año, revistas en las que se publicó, idioma de las publicaciones, autores, instituciones y países de coautoría. Resultados: Se identificaron 380 artículos, 333 de los cuales (87,6\%) se registraron en el período 2006-2010. Fueron citados

*Recibido el 12 de abril de 2012 y aceptado para publicación el 11 de mayo de 2012.

Los autores no declaran conflictos de interés.

Correspondencia: Dr. Javier Moraga C.

Casilla 54 D. Temuco, Chile.

Javier.moragac@gmail.com 
en 1.946 oportunidades (Factor de Impacto de 5,12 e índice-h de 21). Los artículos fueron publicados en Rev Chil Cir (33,4\%), Obes Surg (8,15\%) y World J Surg (3,6\%). Las instituciones con mayor representación fueron Universidad de Chile (33,9\%), Pontificia Universidad Católica de Chile (21,0\%) y Universidad de La Frontera (8,4\%). Conclusión: La producción científica de la cirugía chilena ha mostrado un importante ascenso desde el inicio del período en estudio hasta la fecha. De la misma forma, se observa un incremento de las citaciones de estos artículos.

Palabras clave: Procedimientos quirúrgicos, bibliometría, ISI Web of knowledge, cirugía.

\section{Introducción}

La finalidad de las revistas biomédicas es ser órgano de difusión, y por ende divulgar el conocimiento científico ${ }^{1}$. Los análisis bibliométricos nacen como una herramienta de medición de la literatura que permite observar desde un punto de vista crítico el desarrollo de la actividad científica en diversas áreas ${ }^{2}$. Asimismo, estos análisis permiten caracterizar la producción científica de personas, instituciones y naciones, tanto en términos cuantitativos como cualitativos $^{3,4}$.

Las medidas que presentan mayor importancia dentro de los estudios bibliométricos son la productividad y el factor de impacto (FI). La productividad se puede definir como la cantidad de artículos publicados y el FI como el cociente entre el número de citas de un año en particular a los artículos publicados durante los dos años previos y el número de dichos artículos $^{5,6}$. Existen índices diseñados para sintetizar ambas medidas; de ellos, el factor $\mathrm{H}$ es el más utilizado y corresponde al valor de " $n$ publicaciones que tengan al menos n citas"

Para examinar la productividad y el impacto de una investigación -además de lo anterior- se crearon diferentes indicadores de indización. Uno de los más conocidos es la inclusión en la base de datos Science Citation Index (SCI), la que se caracteriza por estándares de excelencia que la vuelven altamente selectiva, es en esta base de datos donde se incluyó el año 2009 a la Revista Chilena de Cirugía ${ }^{9}$. Es a partir de la información recabada en esta base de datos que se realizará el siguiente análisis bibliométrico, ya que nos parece que es de gran utilidad contar con este tipo de estudios para conocer la realidad de la investigación de la cirugía chilena y así poder evaluar su evolución e identificar sus fortalezas y debilidades.

Nuestro objetivo es definir el perfil bibliométrico de las publicaciones ISI relacionadas con la cirugía chilena en el período 2001-2010, en la base de datos ISI Web of Knowledge de Thomson Reuters.

\section{Material y Método}

Diseño: estudio bibliométrico.

Población a estudio: todas las publicaciones relacionadas con la cirugía chilena indizadas en la base de datos Science Citation Index Expanded (SCI-E) de Thomson Reuters, entre los años 2001 y 2010 (ambos incluidos).

Maniobra: para la selección de los artículos, se realizó una revisión de las publicaciones relacionadas con la cirugía chilena -en revistas indizadas bajo el tópico de cirugía- en el período antes señalado en la plataforma ISI Web of Knowledge de Thomson Reuters, en la cual se aplicaron filtros por país (Chile), por tema (cirugía) y por año (2001-2010). Así, resultó el siguiente algoritmo de búsqueda: $\mathrm{CU}=\mathrm{CHILE}$ AND SO = (SURGERY $)$ AND PY = 2001-2010.

Variables: de los artículos obtenidos a partir de la búsqueda, se utilizaron las siguientes variables: número de publicaciones y de citaciones de los artículos chilenos por años (impacto), revistas en las que se publicó, idioma de las publicaciones, número de publicaciones por autor, instituciones y países de coautoría con el fin de describir la evolución de las publicaciones chilenas en los últimos 10 años.

\section{Definiciones}

Número de publicaciones: es la suma de todos los artículos de la cirugía chilena publicados en revistas ISI, durante el período en estudio.

Citaciones de los artículos chilenos: número de referencias utilizadas en artículos, en las cuales a lo menos un autor presenta nacionalidad chilena.

Número de publicaciones por autor: suma de todas las publicaciones en que haya participado como autor, independientemente del lugar de autoría en que él se encuentre.

Instituciones: corresponden a las instituciones a las cuales se encuentran afiliados los autores de los artículos estudiados.

Países de coautoría: países a los cuales corresponde la nacionalidad de otros autores incluidos en los artículos antes mencionados.

Plan de análisis: se aplicó estadística descriptiva de las variables con cálculos de promedio, desviación estándar y frecuencias. Se realizó un análisis comparativo por quinquenios de la variable número de publicaciones con el objetivo de facilitar el análi- 
sis del lector respecto de esta variable. La tabulación y presentación de los datos se realizó con el software MS Excel 2003.

\section{Resultados}

Para el período y en la base de datos estudiada se identificaron 380 artículos, con un promedio anual de 38. Para el primer quinquenio, el promedio anual fue de 15, y para el segundo el promedio anual fue de 60,6 (Figura 1).

El total de artículos encontrados fueron citados en 1.946 oportunidades. El impacto para el total del período fue de 5,12 con un índice $\mathrm{H}$ de 21 . El factor de impacto para cada año se observa en la Tabla 1.

En relación a las revistas en las cuales se realizaron estas publicaciones, destacan: Rev Chil Cir, Obes Surg y World J Surg, con un 33,4\%, 8,2\% y $3,6 \%$ respectivamente. En la Tabla 2 se puede apreciar las diez revistas en las que más se publicó con sus respectivos porcentajes del total.

El idioma del 65,3\% de los artículos fue inglés, seguido por un $34,2 \%$ y un $0,5 \%$ para el español y el alemán respectivamente.

Los autores más destacados durante este período son Csendes A, Braghetto I y Burdiles P. Los diez autores más destacados de este período y su número de publicaciones se resumen en la Tabla 3.

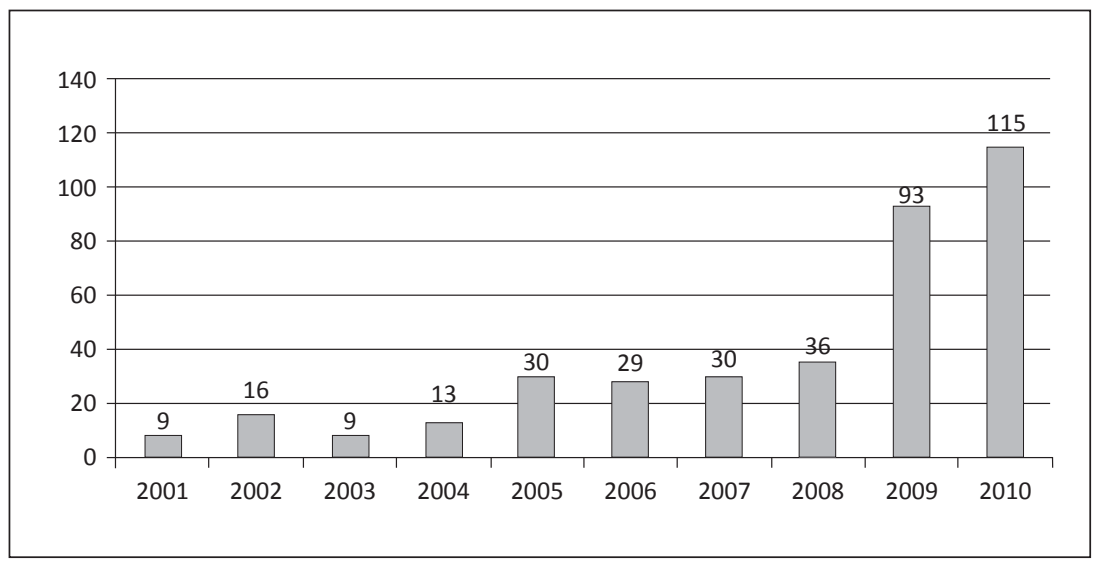

Figura 1. Número de publicaciones ISI chilenas en revistas quirúrgicas por año, 2001-2010.

Tabla 1. Factor de impacto y número de citas de las publicaciones chilenas en revistas quirúrgicas ISI, 2001-2010

\begin{tabular}{|ccc|}
\hline Año & $\begin{array}{c}\text { Factor de } \\
\text { impacto }\end{array}$ & Citas \\
\hline 2001 & 1,040 & 26 \\
\hline 2002 & 0,954 & 21 \\
\hline 2003 & 1,217 & 28 \\
\hline 2004 & 1,080 & 27 \\
\hline 2005 & 1,409 & 31 \\
\hline 2006 & 2,046 & 88 \\
\hline 2007 & 2,694 & 159 \\
\hline 2008 & 1,983 & 117 \\
\hline 2009 & 1,969 & 130 \\
\hline 2010 & 1,162 & 150 \\
\hline
\end{tabular}

Tabla 2. Ranking de revistas quirúrgicas ISI, con publicaciones chilenas, 2001-2010

\begin{tabular}{|lcc|}
\hline Revista & $\begin{array}{c}\mathbf{n} \\
\text { publicaciones }\end{array}$ & $\begin{array}{c}\text { \% } \\
\text { publicaciones }\end{array}$ \\
\hline Revista Chilena de Cirugía & 127 & $33,4 \%$ \\
Obesity Surgery & 31 & $8,2 \%$ \\
\hline World Journal of Surgery & 14 & $3,7 \%$ \\
\hline Arthroscopy-The Journal of Arthroscopic & 12 & $3,2 \%$ \\
and Related Surgery & 12 & $3,2 \%$ \\
\hline Journal of Gastrointestinal Surgery & 8 & $2,1 \%$ \\
Surgical Laparoscopy Endoscopy \& & & \\
Percutaneous Techniques & 8 & $2,1 \%$ \\
\hline Transplantation Proceedings & 7 & $1,8 \%$ \\
\hline Annals of Surgery & 7 & $1,8 \%$ \\
\hline Journal of Pediatric Surgery & 7 & $1,8 \%$ \\
\hline Journal of Surgical Oncology & 147 & $38,7 \%$ \\
\hline Otras & 380 & $100 \%$ \\
\hline Total & & \\
\hline
\end{tabular}


Tabla 3. Ranking de autores con publicaciones en revistas quirúrgicas ISI y total de publicaciones ISI, 2001-2010

\begin{tabular}{|lcc|}
\hline Autor & $\begin{array}{c}\text { n publicaciones en } \\
\text { revistas quirúrgicas }\end{array}$ & $\begin{array}{c}\text { n total } \\
\text { publicaciones }\end{array}$ \\
\hline Csendes, A. & 50 & 90 \\
Braghetto, I. & 25 & 39 \\
\hline Burdiles, P. & 22 & 40 \\
Burgos, Am. & 20 & 23 \\
\hline Manterola, C. & 18 & 33 \\
\hline Escalona, A. & 17 & 24 \\
\hline Korn, O. & 17 & 30 \\
Beltrán, Ma. & 14 & 19 \\
\hline Boza, C. & 14 & 21 \\
\hline Smok, G. & 14 & 43 \\
\hline
\end{tabular}

En cuanto a las instituciones, destacan: Universidad de Chile (33,9\%), Pontificia Universidad Católica de Chile (21\%) y Universidad de La Frontera $(8,4 \%)$. Las diez instituciones más destacadas durante el período en estudio se observan en la Tabla 4.

En términos de cooperación internacional, los países de coautoría en orden de importancia son EE.UU (7,8\%), Brasil (3,6\%), Italia (2,6\%) y España $(2,6 \%)$. Los diez países de coautoría más destacados se pueden observar en la Tabla 5.

\section{Discusión}

A nivel regional y mundial son pocas las personas dedicadas a la realización de estudios bibliométricos. Chile no es la excepción, encontrándose sólo algunas investigaciones de grupos como MINCIRtendientes a valorar calidad metodológica de estudios sobre terapia publicados en la Revista Chilena de Cirugía, asociación con calidad de artículos similares publicados en revistas extranjeras e incremento de la visibilidad de la Revista Chilena de Cirugía a partir de su indización en SciELO ${ }^{1,10}$.

En esta primera publicación se intenta realizar un estudio bibliométrico de carácter descriptivo de la realidad de la cirugía chilena durante la primera década de este siglo. La intención -a pesar de las limitaciones que pueda presentar el estudio- es conocer el número de publicaciones y de citaciones de los artículos chilenos por años (impacto), revistas en las que se publicó, idioma de las publicaciones, número de publicaciones por autor, instituciones y países de
Tabla 4. Ranking de instituciones con publicaciones en revistas quirúrgicas ISI, 2001-2010

\begin{tabular}{|lc|}
\hline Institución & $\begin{array}{c}\text { \% de } \\
\text { publicaciones }\end{array}$ \\
\hline Universidad de Chile & 33,9 \\
\hline Pontificia Universidad Católica de Chile & 21,0 \\
\hline Universidad de La Frontera & 8,4 \\
\hline Clínica Las Condes & 3,4 \\
\hline Universidad de Valparaíso & 3,4 \\
\hline Hospital Universitario (U. de Chile) & 3,2 \\
\hline Clínica Alemana & 2,6 \\
\hline Universidad del Desarrollo & 2,6 \\
\hline Universidad Católica del Norte & 2,4 \\
\hline Universidad de Concepción & 2,4 \\
\hline
\end{tabular}

Tabla 5. Ranking de países en coautoría con publicaciones chilenas en revistas quirúrgicas ISI, 2001-2010

\begin{tabular}{|lcc|}
\hline País & $\begin{array}{c}\text { n de publicaciones } \\
\text { en coautoría }\end{array}$ & $\begin{array}{c}\text { \% de publicaciones } \\
\text { en coautoría }\end{array}$ \\
\hline USA & 30 & 7,9 \\
Brasil & 14 & 3,7 \\
Italia & 10 & 2,6 \\
España & 10 & 2,6 \\
Alemania & 7 & 1,8 \\
Argentina & 5 & 1,3 \\
Francia & 4 & 1,1 \\
Inglaterra & 3 & 0,8 \\
Irlanda & 3 & 0,8 \\
Bélgica & 2 & 0,5 \\
Otro & 25 & 6,6 \\
\hline Total & 113 & 29,7 \\
\hline
\end{tabular}

coautoría; datos que creemos serán de utilidad a los autores nacionales para conocer el escenario nacional actual, incrementar su productividad científica en aquellos que poseen pocas publicaciones y servir de estímulo para aquellos que presentan un nivel de productividad mayor.

Es importante destacar que las revistas al momento de ser indizadas a ISI, quedan generalmente bajo 
un solo tópico como puede ser por ejemplo cirugía, cardiología u otro; el presente estudio fue realizado sólo con revistas bajo el tema de cirugía, por lo cual algunos autores podrían, eventualmente, ver disminuida falsamente su productividad personal. Además existe variación al momento de ingresar los nombres de autores de origen español a las bases de datos biomédicas, lo que puede también generar subestimaciones en el número de publicaciones ${ }^{11}$. Los diez autores más destacados, el número de sus publicaciones en revistas quirúrgicas y el total de sus publicaciones para el período señalado puede observarse en la Tabla 3.

Se puede observar en la investigación un importante ascenso, desde el inicio del período en estudio hasta la fecha, de la productividad científica de la cirugía chilena, lo que también ocurre con las citaciones de estos artículos. El hecho antes mencionado puede deberse a lo menos a dos situaciones: la primera es que exista un aumento real de la producción de artículos científicos por parte de los autores nacionales y que estos sean publicados además en revistas indexadas a ISI; la segunda es que sea debido a la indización de la Revista Chilena de Cirugía a la base de datos de Thomson Reuters, sin que esto haya significado un aumento real de la cantidad de publicaciones de los autores nacionales con respecto a períodos anteriores. $\mathrm{Al}$ parecer, la segunda posibilidad es la que toma mayor preponderancia en este caso, lo que puede interpretarse como un llamado de atención para el mundo quirúrgico chileno, tanto del medio universitario como asistencial, para intentar aumentar la productividad científica y de esta forma incrementar la generación de conocimiento a nivel nacional.

\section{Referencias}

1. Manterola C. Respecto de la calidad metodológica de los artículos que se publican en las revistas biomédicas. Rev Chil Cir. 2005;57:449-50.

2. Vial M, Manterola C, Pineda. V Revista Chilena de Cirugía: Índice de publicación según procedencia de los artículos. Rev Chil Cir. 2008;60:41-5.

3. Abramo G, Ciriaco A, Di Costa F. National research assessment exercises: a comparison of peer review and bibliometrics rankings. Scientometrics 2011. En prensa.

4. Poletto VC, Faraco IM. Bibliometric study of articles published in a Brazilian journal of pediatric dentistry. Braz Oral Res. 2010;24:83-8.

5. Chattopadhyay A. How useful is journal impact factor? Indian J Dent Res. 2009;20: 246-8.

6. Manterola C, Pineda V, Vial M, Losada H. ¿Es el factor de impacto un índice apropiado para determinar el grado de evidencia de estudios sobre procedimientos terapéuticos en revistas quirúrgicas? Cir Esp. 2005;78:96-9.

7. Dorta-González P, Dorta-González MI. Central indexes to the citation distribution: a complement to the h-index. Scientometrics 2011: En prensa.

8. Hirsch JE. An index to quantify an individual's scientific research output. PNAS 2005;102:16569-72.

9. Arribalzaga E. Incorporación al ISI de la Revista Chilena de Cirugía. Rev Chil Cir. 2009;61:313.

10. Manterola C. Revista Chilena de Cirugía indexada en SciELO. Lo relevante y lo que viene por delante. Rev Chil Cir. 2007;59:1-2.

11. Ruiz-Pérez R, Delgado E, Jiménez-Contreras E. Spanish personal name variations in national and international biomedical databases: implications for information retrieval and bibliométrico studies. J Med Libr Assoc. 2002;90:411-30. 